\title{
LA PHILOLOGIE ROMANE EST-ELLE CAPABLE DE RELEVER LES DÉFIS DU PRÉSENT?
}

Le présent article se propose d'esquisser d'abord les grandes lignes de l'évolution de la philologie romane entendue comme discipline scientifique et comme élément de structure de l'université polonaise. La seconde partie constitue une étude de cas qui illustre comment cette évolution s'est répercutée sur mes choix et mon parcours universitaire.

\section{DISCIPLINE ET INSTITUTIONS}

L'existence et le fonctionnement de la philologie romane sont étroitement liés à l'histoire de l'institution universitaire en Pologne. En 2017, le plus ancien institut de philologie romane, celui de l'Université Jagellonne de Cracovie, a fêté son $125^{\mathrm{e}}$ anniversaire. Ceux de l'Université de Poznań, de Varsovie et de l'Université Catholique de Lublin ont été inaugurés en même temps que l'université de la Pologne indépendante, en 1919. À l'origine - mais c'est encore parfois le cas aujourd'hui —, la dénomination de philologie désignait une entité universitaire vouée aux études de lettres. La première division de ce vaste ensemble distingue la philologie polonaise et les philologies étrangères : allemande (germanique), anglaise, russe, etc. La philologie romane fait partie de cet ensemble, mais sa nature particulière exige des éclaircissements, ne serait-ce que du fait de sa dénomination ambiguë. 


\subsection{HÉRITAGE ALLEMAND}

Le terme couramment employé dans nos universités pour désigner l'étude des littératures d'une zone linguistique et culturelle relève de la tradition académique allemande, dont le système universitaire polonais est en grande partie tributaire. Le terme " philologie », suggéré par le français, langue de cette spécialité, signifie étude d'une langue à partir de documents écrits. Au sens restreint, cette science vise à rétablir le contenu original de textes connus par plusieurs sources, c'està-dire à sélectionner le texte le plus authentique possible, à partir de manuscrits, d'éditions imprimées ou d'autres sources disponibles (citations, voire inscriptions anciennes). La démarche implique le rétablissement du «meilleur » texte en corrigeant les sources existantes. Il s'agit donc d'une combinaison de critique littéraire et linguistique qui se rapporte à l'étude de la langue conservée dans les plus anciens textes.

Dans l'acception allemande - et par conséquent polonaise -, le terme a un sens beaucoup moins précis et désigne l'étude de la langue et de la littérature d'une zone linguistique : française, anglaise, allemande, etc., sans insister sur le caractère historique de l'objet d'études, même si des éléments de grammaire historique et d'étude d'anciens textes figurent dans les programmes d'enseignement. Le terme de «philologie romane » sert aussi à désigner les structures (instituts, départements, chaires) responsables de l'organisation des études, qui regroupent des enseignants et chercheurs ayant en premier lieu le français comme instrument de travail et objet d'investigations. Dans de nombreux cas, ces étiquettes restent d'actualité en Pologne. La divergence d'acceptions cause cependant parfois des malentendus chez les Français, étonnés de voir que les programmes d'études des chaires de philologie romane polonaises réservent une large part à l'étude du français et de la littérature contemporains. Il faut noter aussi que dans notre nomenclature universitaire, ces unités portent le nom de " néophilologies », qui les démarque de la philologie classique destinée à l'étude des langues mortes (grec, latin) d'une part, et de la philologie polonaise de l'autre.

Une deuxième série de malentendus prend sa source dans l'acception de l'épithète « romane » qui, en français, renvoie à l'émergence du français et aux plus anciens stades de l'évolution de la langue. Dans la pratique des universités polonaises, il s'agit avant tout des études françaises, et le cursus universitaire comporte l'apprentissage d'une autre langue romane (italienne, espagnole, portugaise, roumaine) en un nombre d'heures réduit, et c'est cela qui justifie l'emploi de l'adjectif « romane ». Il en va de même pour la philologie germanique où la prédominance de l'allemand éclipse d'une certaine façon les autres langues germaniques, exception faite de l'anglais qui est étudié dans le cadre d'une philologie distincte. 


\subsection{UNIVERSITÉS ET POLITIQUE}

La situation des départements de philologie au sein des universités polonaises, par exemple le recrutement des étudiants et, par conséquent, des enseignants, mais aussi les axes de recherches, les relations avec les pays et les cultures de référence, etc., ont toujours été étroitement liés à la situation politique ${ }^{1}$. Ce fut surtout le cas sous le régime communiste, de la fin des années 1940 aux années 1990. La place des philologies au sein de l'université et, par la force des choses, le financement des recherches, le nombre de postes ouverts et d'étudiants admis, se faisaient au gré de la température des relations politiques de la Pologne avec les pays respectifs. Ainsi, les départements de philologie russe étaient privilégiés sur tous les plans. Ceux de philologie anglaise s'orientaient vers la littérature de Grande-Bretagne, les États-Unis étant, dans les années 1950-1970, considérés comme l'ennemi juré de tout le bloc soviétique ; les philologies germaniques se référaient uniquement à la République Démocratique Allemande, et tout soutien matériel offert par les institutions universitaires ou diplomatiques de la République Fédérale devait être rejeté ou, du moins, occulté. Il va sans dire que la France était le point de référence presque exclusif de la philologie romane, dont la situation reflétait les relations politiques polono-françaises, marquées par des durcissements et des dégels successifs. À titre d'exemple, on peut citer la suspension du recrutement d'étudiants à la philologie romane de Wrocław dans les années 1951-1952 puis dans les années 1984-1985, ou à la chaire de philologie romane de l'Université Catholique de Lublin en 1961, prélude à sa fermeture en 1964. Les deux cas étaient motivés uniquement par des raisons politiques et illustrent de manière éloquente la mainmise du pouvoir communiste sur les universités polonaises.

Le dégel du début des années 1970 a freiné le déclin des philologies romanes et a inversé cette tendance. C'est au début de la décennie, en 1973, qu'on a créé deux nouveaux départements de philologie romane : un à l'Université de Silésie, et un autre à l'Université Marie Curie-Skłodowska de Lublin. La même année, les philologies romanes de l'Université catholique de Lublin et de l'Université de Łódź ont été autorisées à reprendre leurs activités pédagogiques. La chaire de philologie romane de l'Académie Pédagogique de Cracovie (aujourd'hui Université Pédagogique) a été créée en 1978. Il faut noter aussi la croissance spectaculaire du nombre d'élèves de français dans les lycées et la création de plusieurs lycées bilingues franco-polonais à la même époque ${ }^{2}$. Et une fois encore ce tournant a eu des motivations politiques : les autorités nouvellement installées comptaient resserrer les liens de la Pologne avec l'Occident afin d'obtenir des crédits pour faire sortir le pays de la situation économique désastreuse dans laquelle il s'était retrouvé à la

${ }^{1}$ D. Jarosz, M. Pasztor, Stosunki polsko-francuskie 1944-1980, Polski Instytut Spraw Międzynarodowych, Warszawa 2008.

2 Ibidem, pp. 359-360. 
fin des années 1960. Les sympathies francophones du secrétaire général du parti communiste Edward Gierek qui, dans sa jeunesse, avait travaillé comme mineur en France et en Belgique, expliquent en grande partie ce regain de francophilie.

Cette ouverture sur l'Europe francophone s'est cependant accompagnée de modifications considérables dans les programmes d'études. Les autorités de l'éducation nationale ont fixé un objectif utilitaire pour les études philologiques : désormais, elles devaient former uniquement des enseignants de langues pour les lycées (car à l'époque, les langues occidentales n'étaient enseignées que dans le secondaire), et la durée des études a été réduite à 4 ans, au détriment des cours de littérature et de théorie linguistique.

Toutefois, la perception des études philologiques, surtout en ce qui concerne les philologies occidentales, dont la romane, allait à l'encontre du pragmatisme imposé par les autorités. J'ai justement fait partie de ces nombreux étudiants qui ont peuplé les départements de français nouvellement créés, et qui n'aspiraient aucunement à la carrière pédagogique, mais envisageaient plutôt de trouver du travail dans le secteur commercial ou dans des institutions culturelles. Dans les années 1950-1970, la France jouissait d'un prestige culturel considérable. Les élites intellectuelles et les universitaires polonais étaient au courant des réussites françaises dans ce domaine, malgré le rideau de fer, d'ailleurs moins hermétique en Pologne que dans les autres pays satellites de l'URSS. De surcroît, le prestige de la France remontait aux traditions de francophonie et de francophilie des milieux intellectuels et des élites polonaises d'avant-guerre. Les acquis français dans la recherche en lettres et en sciences humaines constituaient un paradigme scientifique et une source d'inspiration pour les chercheurs polonais. À titre d'exemple, on peut mentionner le rayonnement des théories venues de Paris et très bien diffusées dans les milieux académiques polonais grâce aux traductions. Ainsi, la revue d'études littéraires la plus ancienne, Pamiętnik Literacki, qui avait une influence considérable sur les recherches littéraires en Pologne, contenait une rubrique « traduction » où étaient publiées des études théoriques et méthodologiques d'auteurs occidentaux célèbres. Or, les traductions du français y occupaient une place considérable, venant en deuxième position après l'anglais. Il en va de même pour une autre revue, plus jeune, mais presque aussi prestigieuse, Teksty, et pour son héritière Teksty Drugie ${ }^{3}$. Pour les jeunes qui entraient à l'université, être étudiant de philologie romane offrait la possibilité d'accéder à une culture prestigieuse et donnait le sentiment de faire partie d'une élite. Ce sentiment était d'autant plus justifié que l'adhésion à cette élite était soumise à un concours d'entrée difficile, le nombre de candidats excédant de plusieurs fois celui des places disponibles.

${ }^{3}$ E. Skibińska, « La traduction dans les revues comme voie de circulation des voix théoriques : L'exemple des revues polonaises Pamiętnik Literacki (1956-1994), Teksty (1972-1981) et Teksty Drugie (1990-2010) », [dans :] Translating the Voices of Theory = La Traduction des voix de la théorie, I. Génin, I. Klitgård (dir.), Éditions québécoises de l'œuvre, Montréal 2015, pp. 113-134. 
La recherche constitue un autre volet important des activités des départements de philologie romane. Elle suivait les grandes tendances en vigueur à l'époque dans le domaine des lettres, aussi bien en Pologne qu'ailleurs. La recherche pratiquée au sein des instituts philologiques se scindait en deux grandes disciplines : études littéraires et linguistiques, bien séparées et parfois même rivales. De plus, en ce qui concerne les études littéraires, elles se concentraient sur de grands auteurs reconnus ou, de manière générale, des auteurs décédés. Les difficultés majeures auxquelles se heurtaient les chercheurs augmentaient du fait de la difficulté d'accès aux sources, et surtout, aux ouvrages critiques et articles de revues spécialisées, les collections des bibliothèques universitaires polonaises étant lacunaires. Les philologies romanes se tournaient vers la France non seulement en raison de l'origine des auteurs étudiés, mais aussi parce qu'elle pouvait leur offrir une possibilité de consulter les publications indispensables. De plus, elle proposait à l'époque des modèles théoriques et méthodologiques de recherche largement suivis dans le monde entier. Étant donné les difficultés politiques (impossibilité de sortir du pays) et économiques (le système économique et monétaire polonais était incompatible avec celui d'Occident), c'étaient surtout les bourses offertes par le gouvernement français qui permettaient aux universitaires polonais de poursuivre leurs recherches. La France était d'ailleurs le plus généreux de tous les pays occidentaux, à en juger d'après le nombre de bourses offertes aux chercheurs et enseignants polonais. C'est ainsi que les liens entre les philologies romanes polonaises et la France se sont resserrés et que la synonymie entre philologie romane et études françaises est devenue presque parfaite.

\subsection{OUVERTURES}

\subsubsection{NOUVEAUX CHAMPS DE RECHERCHE}

Les années 1970 apportent également des changements considérables dans le domaine de la recherche. Ils sont bien perceptibles dans l'apparition de nouveaux champs d'investigation et de nouvelles approches. Les cloisons entre les disciplines sont devenues moins étanches — dans les années 1960 déjà, le structuralisme a fait découvrir l'intérêt des analyses intrinsèques du texte littéraire, dont l'interprétation ne devait pas tenir compte des facteurs relevant de l'histoire littéraire, tels le contexte intellectuel dans lequel l'œuvre a paru, ou la biographie de l'auteur, dont l'œuvre serait un reflet. Cette tendance a rapproché les études littéraires de la linguistique. Par ailleurs, le champ d'intérêt des linguistes s'est élargi : il a dépassé les limites de la phrase pour inclure le discours réellement produit et ses actualisations (les textes), ce qui a noué progressivement le dialogue entre les études littéraires et la linguistique, jusqu'ici séparées. Après la domination de l'approche structuraliste et de la grammaire générative, c'est la grammaire cognitive qui s'est développée, établissant un nouveau paradigme scientifique 
avec le postulat d'étudier la langue comme élément indissociable de son vaste contexte culturel. Les études culturelles développées dans le milieu anglo-saxon ont rayonné aussi dans le domaine des études francophones. En même temps, le concept même de culture s'est « démocratisé » pour désigner, outre les arts, les lettres et les sciences, les modes de vie, les lois, les systèmes de valeurs, les traditions et croyances, et même des artefacts. Cet éclatement de la notion de culture et l'égalité dans la façon d'étudier ses différentes manifestations se sont substitués peu à peu à la notion de civilisation française, connotée d'un prestige vénérable et conservant sa valeur de référence. La littérature, composante la plus raffinée de la civilisation, a perdu sa place privilégiée au profit des phénomènes culturels quotidiens, moins nobles, ce qui a contribué à un déplacement ou, du moins un élargissement, du centre d'intérêt des chercheurs.

\subsubsection{FRANCOPHONIE}

L'évolution sociale et politique a contribué également à une redéfinition des objectifs de recherches. Le cas de la France, point de référence des chercheurs en philologie romane de Pologne, en est un excellent exemple. D'une part, elle a cessé progressivement d'être perçue comme une culture monolithique circonscrite aux limites de l'hexagone et marquée par ses longues traditions vénérables. Cette prise de conscience a correspondu à la remise en cause partielle de la politique d'assimilation pratiquée en France. Le projet de ramener tous les habitants du pays, indépendamment de leur origine, au modèle unique de la culture hexagonale s'est avéré impossible à réaliser. Ce modèle a cédé la place à un autre, celui de l'intégration, qui reconnaît la valeur des cultures venues d'ailleurs et leur impact sur la culture française. Dans ce contexte est né l'intérêt pour les littératures des autres pays où le français est en usage et dont les œuvres littéraires sont rédigées en français. En dehors des régions voisines de la France, la Wallonie et la Suisse Romande, dont les littératures avaient déjà leur petite place dans l'enseignement et la recherche de certains départements de philologie romane polonais, plusieurs chercheurs se sont concentrés sur les littératures et cultures des pays et régions lointains, tels le Québec, le Maghreb, Haïti, etc. À cela se sont ajoutées les activités de plus en plus visibles de la Francophonie institutionnelle devenue un acteur important dans la vie culturelle et universitaire mondiale et qui a fait découvrir ou favorisé la promotion des cultures des pays francophones.

Par ailleurs, dans les années 1980, la France a redéfini les objectifs de sa politique linguistique, qui s'est distanciée par rapport aux valeurs culturelles traditionnelles. Elle s'est tournée délibérément vers la promotion des réussites technologiques, économiques et autres, par le biais des langues de spécialité. Ce tournant s'est fait au détriment des valeurs culturelles et littéraires traditionnellement mises en vedette. Par conséquent, le soutien du gouvernement français aux philologies romanes polonaises a diminué. 


\subsubsection{TRANSFORMATION POLITIQUE}

Le tournant politique majeur de 1989 a remis la Pologne sur de nouveaux rails, avec la liberté de circulation, la chute du rideau de fer et, enfin, l'entrée du pays dans l'Union européenne. Ce nouveau contexte a profondément modifié la situation des universités polonaises et, par conséquent, celle des départements de philologie romane. Avant tout, la plupart des entraves d'ordre politique dans les relations avec la communauté scientifique internationale ont disparu, ce qui a ouvert aux chercheurs polonais des possibilités de participer à part entière dans la vie universitaire européenne et mondiale. Les philologies romanes de Pologne ont réagi à ces changements multiples. D'une part, on a assisté à l'amplification des études des autres langues et littératures romanes (italienne, espagnole, portugaise, roumaine). Dans certains cas, cela a provoqué l'éclatement des départements de philologie romane et la création de structures (instituts, départements, chaires) autonomes vouées aux études italiennes, ibériques, etc. Cette tendance a contribué à diminuer le poids du français au sein des unités existantes. D'autre part, l'enseignement de la littérature française a cédé de plus en plus de place à l'étude des langues, littératures et cultures des autres pays francophones. Des chercheurs polonais ont pu bénéficier du système de bourses mises à la disposition des chercheurs des pays d'Europe centrale et orientale, leur permettant d'effectuer des séjours de recherches dans différents pays ou auprès de différentes institutions francophones ${ }^{4}$. Le fait que la Pologne ait retrouvé sa place dans la vie scientifique internationale explique également le développement de la recherche en traductologie menée au sein des départements de linguistique, qui a acquis aujourd'hui une importance égale à celle des études linguistiques théoriques.

\subsubsection{MONDIALISATION}

Enfin, la révolution informatique et l'avènement des nouvelles technologies ont eu un fort impact sur le monde universitaire. Les facilités qu'offre Internet, la rapidité des contacts entre les universités et les chercheurs ont contribué à donner une nouvelle dynamique aux recherches, aux échanges, à la formulation des projets de recherche qui engagent désormais plusieurs universités ou groupes de recherche. La numérisation des ressources a rendu plus accessible une grande partie des sources et des publications qu'on ne pouvait consulter autrefois que dans les bibliothèques. Ces facilités ont encouragé de nombreux chercheurs à adhérer à des équipes universitaires internationales, à formuler des projets de recherches transversales, comparatives, englobant des œuvres ou auteurs issus de différents pays francophones et même excédant les limites de la francophonie. Ce resserrement

\footnotetext{
${ }^{4}$ La Pologne a adhéré à la Francophonie en 1997.
} 
des relations a eu pour conséquence des rapprochements tant des thèmes de recherche que des approches méthodologiques.

La mondialisation, intimement liée à la révolution informatique, a également exercé une influence considérable sur la recherche. Avant tout, il faut noter la remise en cause des notions de nation et d'identité nationale, indissociablement liées aux axes de recherche traditionnellement associés aux recherches littéraires. L'abolition des cloisonnements entre les pays et les cultures, qui a abouti à l'uniformisation des cultures et, pour les individus, à l'apparition des identités multiples, en a été la conséquence logique. La facilité de déplacement des hommes et des idées, encouragée par le rôle grandissant des médias et des nouvelles technologies, a fait apparaître de nouveaux phénomènes culturels. Les migrations humaines forment de nouvelles attitudes et de nouveaux types d'identité qui rentrent désormais dans le domaine des recherches universitaires. Dans le cas de la littérature, par exemple, il faut mentionner le métissage culturel qui impose un réexamen de la notion de littérature nationale suite aux mélanges d'éléments de diverses provenances dans l'œuvre des auteurs qui vivent à cheval sur deux ou même plusieurs langues et cultures. Ces éléments sont perceptibles malgré l'adoption du français comme langue d'écriture. Les textes métissés intègrent plus systématiquement des éléments d'autres langues, à travers lesquels intervient une autre culture, et qu'on ne peut cependant pas identifier comme des écarts par rapport à la culture française. Il s'agit d'une culture différente, dans la plupart des cas déterritorialisée. Un tel objet de recherche implique l'abandon des méthodologies éprouvées et la nécessité d'en proposer de nouvelles. Il ne s'agit plus uniquement de prise en compte de théories importées d'ailleurs que de Paris, mais de théories susceptibles d'englober des phénomènes culturels de portée très générale, telles par exemple les théories postcoloniales et postmodernes ${ }^{5}$. Les bibliographies placées à la fin des ouvrages publiés par les romanistes polonais, qui jusqu'ici ne contenaient que rarement des ouvrages écrits en une autre langue que le français, s'enrichissent de mentions d'ouvrages relevant des autres traditions scientifiques. La mise en place de nouvelles sources de financement des recherches et d'un système de bourses internationales ont accru considérablement les possibilités d'effectuer des séjours de recherches dans différents pays ou auprès de différentes institutions francophones.

Cet éclatement embrouille l'identification de la philologie romane en tant que discipline scientifique définie à partir de son objet et de sa méthodologie. La multiplicité des objets d'études, les différentes approches théoriques qui tendent vers le syncrétisme, les phénomènes tels que la littérature migrante, qui se manifeste sous des formes analogues dans le domaine des langues anglaise et française,

${ }^{5}$ D'ailleurs, les écrits théoriques des figures de proue du postmodernisme, comme Jean Baudrillard, Gilles Deleuze ou Félix Guattari, ont acquis une grande audience en Europe après avoir connu d'abord un vif engouement dans les départements américains de Lettres, où s'est constitué un corpus de théories philosophiques englobé sous la dénomination de French Theory. 
contribuent à remettre en cause les anciennes divisions disciplinaires basées sur de mêmes approches méthodologiques. L'évolution de la recherche en philologie romane suit celle des changements généraux dans le domaine de la recherche en littérature. Fondue dans la réalité complexe de la culture en crise, elle se distingue par le dépassement systématique de toutes les limites de la philologie traditionnelle : depuis l'étude de l'œuvre littéraire jusqu'à celle de l'ensemble syncrétique de phénomènes culturels; depuis une approche méthodologique précise, telle la sémiotique, la mythocritique, etc. jusqu'à l'éclectisme méthodologique dont le système est chaque fois reconstruit et reconfiguré selon le besoin d'une étude précise. Le dialogue des disciplines devient réel, et il mène à la création de nouveaux systèmes et réseaux conceptuels. L'interdisciplinarité devient une règle incontournable.

\section{PARCOURS PERSONNEL}

Dans les chaires de philologie romane, les tendances brossées ci-dessus ont déterminé les choix des chercheurs en littérature. Les changements de paradigmes scientifiques ont fait émerger des vagues successives de jeunes adeptes des études interdisciplinaires qui s'éloignent des recherches philologiques traditionnelles. Toutefois, certains parcours individuels attestent une certaine continuité des préoccupations scientifiques, sans pour autant sombrer dans un conservatisme académique. Mon cas, sans être peut-être très typique, en est un exemple.

Après mes études de philologie romane, je suis entré à l'université en qualité d'assistant, et j'ai été confronté au problème du choix d'une spécialisation, ce qui à l'époque (en 1977), se résumait à la dichotomie linguistique ou études littéraires. Ayant rédigé mon mémoire de maîtrise en littérature, j'ai choisi ces dernières, et il m'a alors fallu choisir également la période littéraire dans laquelle j'allais me spécialiser. J'ai décidé de me consacrer à la littérature française du Moyen Âge, décision qui peut être considérée comme un acte de conformisme envers la tradition philologique. Dans la deuxième moitié des années 1970, en Pologne, cette spécialisation représentait un défi difficile à relever. Tout d'abord, elle impliquait la nécessité de maîtriser l'ancien français, matière première des textes que j'aurais à étudier, or en règle générale, les diplômés de philologie romane n'avaient que quelques notions de la morphologie de la vieille langue. Ensuite, l'accès aux sources et à la documentation posait d'énormes problèmes, vu la pauvreté des bibliothèques universitaires polonaises en ouvrages et revues spécialisées occidentaux, malgré l'aide systématique du gouvernement français. Les chercheurs polonais en la matière étaient également peu nombreux. J'ai heureusement pu compter sur deux professeures, Krystyna Kasprzyk de l’Université de Varsovie et Anna Drzewicka de l'Université Jagellonne de Cracovie, éminentes spécialistes de la littérature médiévale. C'est sous leur tutelle que s'est constitué un groupe 
de jeunes chercheurs de différents centres universitaires polonais. Peu nombreux, mais très motivé, il se réunissait régulièrement et formait un contexte rassurant, sans pour autant pallier tous les manques ressentis. Plusieurs stages de recherche accordés par le gouvernement français m'ont permis d'avancer dans mes recherches et de soutenir ma thèse de doctorat en 1986.

L'intérêt pour le Moyen Âge semble s'inscrire dans la conception traditionnelle de l'université qui, d'après Jorge Luis Borges, doit enseigner ce qui est ancien et étranger au lieu de ce qui est moderne et connu pour éviter de jouer le même rôle que le journalisme. Mais, paradoxalement, le corpus médiéval, lacunaire et difficile à interpréter du fait de son éloignement temporel et de son inscription dans une mentalité spécifique, exige la recherche d'une méthodologie adéquate. L'altérité foncière des œuvres médiévales rend très vite conscient que l'outillage des sciences littéraires élaboré à partir du corpus moderne n'est pas suffisant. Afin de comprendre le sens et la composition des textes, leurs «san » et «matiere $»^{6}$, il me fallait dépasser les limites rassurantes des études littéraires et inclure dans ma réflexion des données historiques et linguistiques. L'approche interdisciplinaire n'était donc pas une question de choix du chercheur, mais s'imposait d'emblée.

Il va sans dire que l'étude d'un corpus constitué de textes médiévaux français impliquait la consultation d'ouvrages critiques produits en France par des universitaires en majorité français. Cette nécessité correspondait étroitement à la recherche des inspirations méthodologiques. Dans mon cas, cette inspiration est venue des études sémiotiques de l'« École de Paris » animée par Algirdas Julien Greimas. Et comme mon corpus était constitué d'œuvres narratives ou lyrico-narratives, le recours à la narratologie française, surtout aux idées de Tzvetan Todorov, Gérard Genette ou Claude Bremond, m'a fourni des outils analytiques très utiles pour découvrir le sens des œuvres. D'autre part, les investigations des historiens de l'École des Annales ont contribué à la reconstitution, dans une certaine mesure, du contexte mental dans lequel les œuvres avaient été composées.

Les « Parisiens » n'ont cependant pas suffi. Contrairement aux études littéraires, la linguistique avait à l'époque la réputation de science exacte offrant un modèle d'investigation marqué de précision et d'objectivité. Or la linguistique générale pratiquée en France et, par imitation, dans les milieux romanistes polonais, présentait une approche trop centrée sur le français contemporain et sur la phrase comme entité maximale. Celle issue du milieu des ethnolinguistes polonais de Lublin, en revanche, dépassait ces limites trop restreintes et proposait une approche de la langue, actualisée dans des textes stéréotypés, répétitifs et hautement prévisibles, comme partie intégrante de la culture. Cette approche correspondait parfaitement à mes interrogations adressées aux textes du Moyen Âge

${ }^{6}$ C'est ainsi que le premier romancier français du XII ${ }^{\mathrm{e}}$ siècle, Chrétien de Troyes, définit les aspects fondamentaux de son Lancelot ou le chevalier de la charette (Chrétien de Troyes, Le chevalier de la charrete, M. Roques (dir.), Champion, Paris 1981, vv 26). 
qui, eux aussi, connaissaient ce phénomène. Ainsi, parti d'un corpus conforme aux préoccupations traditionnelles de la philologie, j'ai été amené à rejoindre de nouvelles orientations, à dépasser les limites disciplinaires et le francocentrisme pratiqué habituellement dans les philologies romanes de Pologne.

La transformation politique survenue en Pologne en 1989 a suscité — je l'ai déjà signalé - un regain d'intérêt pour les langues occidentales, vouées à supplanter l'enseignement obligatoire du russe dans le primaire et le secondaire. Tout à coup, le système scolaire polonais a ressenti un grand besoin d'enseignants de langues occidentales, dont le français. Dans le cadre du soutien à la formation des maîtres d'anglais et de français, un organisme québécois, OXFAM - Québec, s'est implanté dans plusieurs villes du Sud-Est de la Pologne. Amené à coopérer avec les Canadiens en qualité de superviseur universitaire, $j$ 'ai pu prendre conscience de la spécificité de leur culture francophone, formée dans un contexte historique différent de celui de la francophonie européenne et fonctionnant dans un environnement bilingue. Cette prise de conscience a été en même temps celle de l'existence de la francophonie, phénomène linguistique et culturel d'une grande richesse, diversifié et ignoré dans une immense majorité des instituts de philologie romane polonais jusqu'à cette époque. L'importance de cette découverte m'a amené à réorienter mon enseignement et à l'élargir par la prise en compte de la francophonie québécoise, américaine, et de la francophonie en général, au sens culturel et institutionnel. Cette décision a eu une conséquence pour le nouveau champ de recherches, celui de la francophonie américaine, qui venait de s'ouvrir. Tout en continuant ma réflexion sur la littérature et sur la linguistique et tout en utilisant des concepts méthodologiques élaborés par ces disciplines, j'ai entamé des études d'histoire et de la culture des régions francophones du Canada.

Un processus analogue a eu lieu dans différents autres départements de philologie romane en Pologne. Depuis, les recherches en littératures francophones se sont développées de manière spectaculaire et occupent aujourd'hui une place considérable dans plusieurs universités polonaises. Les études de littérature québécoise, par exemple, sont devenues très prisées, ce que prouvent plusieurs thèses dans ce domaine, des publications de haut niveau appréciées par le milieu québéciste international, et des prix prestigieux accordés aux chercheurs polonais. De nouveaux champs de recherche se sont ouverts. En dehors des différentes littératures francophones, notons le succès des thèmes transversaux tels que la littérature migrante, les littératures mineures, etc. Ces nouvelles préoccupations en vogue ont deux traits communs : d'une part elles se concentrent sur des phénomènes littéraires et culturels récents, et d'autre part, elles s'opposent en quelque sorte à la littérature comprise traditionnellement comme liée à un territoire et à une tradition précis.

La tendance à transgresser la vision territorialisée de la culture pour l'appréhender transversalement contribue de manière logique à la disparition des cloisons disciplinaires. Cette dernière se manifeste dans les choix individuels 
des chercheurs, mais aussi, exerce une influence sur la structure de l'université, généralement peu encline aux changements. À titre d'exemple, on peut citer le cas de l'université de Silésie, où la dénomination "Institut de Philologie Romane » a été remplacée en 2007 par «Institut de Langues Romanes et de Traductologie », ce qui correspond mieux aux préoccupations scientifiques et pédagogiques de cette institution. J'ai moi-même commencé à collaborer avec l'Institut des Recherches Interdisciplinaires de l'Université de Varsovie, devenu depuis 2013 la Faculté «Artes Liberales ». Cette institution, désormais bien ancrée dans la structure de l'Université de Varsovie, a fait de la coopération des représentants de différentes disciplines un de ses grands principes. Bien sûr, des philologues y sont représentés (dont deux romanistes). Mais on y trouve également des linguistes, historiens, philosophes, sociologues, spécialistes des études culturelles, et même des représentants de disciplines telles que la psychologie et la biologie. Malgré un nombre conséquent de philologues au sein de la faculté, cette spécialisation ne prend pas le dessus sur les autres disciplines représentées. Grâce à cet équilibre, les activités scientifiques de la faculté donnent des résultats très satisfaisants dans le domaine des recherches inter- ou même transdisciplinaires. Ses projets de recherches, ses différents colloques, journées de travail et publications prônent une approche syncrétique des champs de recherche et des méthodologies. Vu les tendances actuelles dans la recherche en lettres (humanities), on peut aisément deviner que ce type d'institutions dont la Faculté «Artes Liberales » est le premier exemple se multipliera, malgré la lenteur habituelle de l'évolution de l'institution universitaire.

\section{CONCLUSION}

Faut-il en conclure que la philologie en général et la philologie romane en particulier fait partie du passé et que cette discipline va se fondre dans le vaste ensemble pluridisciplinaire du monde postmoderne ? Il y a tout lieu de croire que, malgré l'évolution esquissée ci-dessus, la philologie, entendue comme une approche plutôt que comme une discipline rigide, n'est pas vouée à l'oubli. Même si les nouvelles tendances dans la recherche sont devenues très visibles et attirent l'attention d'un nombre considérable de chercheurs, l'étude des vieux textes n'a pas entièrement disparu des universités et la philologie traditionnelle donne toujours des clés pour les étudier et, par conséquent, pour connaître et comprendre le passé.

La philologie est avant tout une étude des textes. Même si le concept de texte a subi un élargissement considérable - depuis le texte écrit, à travers le texte oral, jusqu'au texte de culture qu'il faut comprendre comme une séquence de signes, pas forcément verbaux, agencés selon les règles d'un système sémiotique 
donné - c'est lui qui est la première donnée empirique soumise à l'examen scientifique, le premier objet des activités pédagogiques. La rigueur analytique que la philologie a érigée en exigence de premier ordre n'a rien perdu de son actualité. Le respect du texte, première condition de la démarche philologique, permet d'éviter les dérapages qui guettent les études interdisciplinaires globalisantes ${ }^{7}$.

Quant aux textes écrits, objet traditionnel des études philologiques, le postulat en vigueur depuis la Renaissance fait rechercher et analyser les textes les plus authentiques possible, donc leur lecture dans les langues d'origine. Le grand intérêt des romanistes pour la traduction, préoccupation par excellence philologique, prouve l'importance de la réflexion sur les langues dans un esprit philologique. Le français, même s'il a beaucoup perdu de son prestige, demeure une langue vivante, langue de communication et de littérature, et c'est une langue plurielle. Tandis que les théories peuvent s'articuler en diverses langues - le cas de la «French Theory » en est le meilleur exemple - , les textes qu'on se propose d'étudier et d'interpréter doivent être lus en version originale. Modifiée, redéfinie, resituée dans son contexte postmoderne, la philologie romane a encore un rôle important à jouer, à condition toutefois d'abandonner sa définition restrictive et de lui assurer une ouverture sur la complexité des cultures tout en préservant ses acquis traditionnels.

\section{IS FRENCH PHILOLOGY READY TO FACE THE CHALLENGES OF TODAY?}

\section{Summary}

The article comprises two sections: in Section One I sketch out the history and the evolution of French philology, understood both as an academic discipline and as an academic/administrative unit within Polish universities, officially known as Departments of French (Philology). In Section Two I reflect on my personal experience of that evolution, as it has affected my professional choices and academic career.

Both meanings of "French philology" (discipline and institution) are rooted in German academic tradition to which the entire system of Polish humanities is indebted. Until the 1990s, French philology was synonymous with French studies, understood as the teaching and the academic study of French language and literature. Like other humanities departments in Poland, French philology departments inevitably functioned under the pressure of current political forces. Yet, French philologists in Poland never lost touch with the world's evolving humanities or the changing scholarly paradigms.

Following the radical political transformation of 1989, traditional French philology in Poland opened up to a whole new range of scholarly fields (literatures and cultures of francophone countries), theories (postmodern and postcolonial studies), and approaches (interdisciplinary scholarship). Thus Polish romanists have joined the international scholarly community.

7 D. Heck, « Problem filologii. Metoda filologiczna dzisiaj? », Litteraria: teoria literatury metodologia — kultura — humanistyka XXXVII, 2009, pp. 7-19. 
In the article, I document these processes, reflecting on my own university career: I started off as a traditional scholar doing research in the literature of French Middle Ages, then moved on to studying Canadian and American Francophone cultures, to eventually become involved in interdisciplinary studies at the Faculty of "Artes Liberales" at the University of Warsaw.

Key words: Roman philology, university, history, methodology. 